\title{
Avaliação de saúde bucal em idosos da Universidade da Maturidade: estudo transversal
}

Oral health assessment in the elderly at the University of Maturity: a cross-sectional study

Evaluación de la salud oral en ancianos en la Universidad de Madurez: estudio transversa

Eduardo Aoki Ribeiro SERA ${ }^{1}$

Neila Barbosa OSÓRIO ${ }^{2}$

Emerson Moura RIBEIRO ${ }^{3}$

Luiz Sinésio SILVA NETO ${ }^{4}$

'Graduado em Odontologia, Mestrando do Programa de Pós-Graduação em Ensino em Ciência e Saúde - PPGECS Universidade Federal do Tocantins - UFT, 77001-090 Palmas-TO, Brasil

${ }^{2}$ Professora Doutora do Programa de Mestrado em Educação e autora do Programa Universidade da Maturidade - UMA Universidade Federal do Tocantins - UFT, 77001-090 Palmas-TO, Brasil

${ }^{3}$ Graduado em Fisioterapia pelo Instituto de Ensino e Pesquisa Objetivo - IEPO, 77021-634 Palmas-TO, Brasil

${ }^{4}$ Professor Doutor do Programa de Mestrado Ensino em Ciências em Saúde e do Curso de Medicina da Universidade Federal do Tocantins - UFT, 77001-090 Palmas-TO, Brasil

\begin{abstract}
Resumo
Introdução: A saúde bucal dos idosos pode ser considerado um problema de saúde pública. Objetivo: O estudo descreve o perfil da saúde bucal dos idosos da Universidade da Maturidade (UMA) do campus de Palmas da Universidade Federal do Tocantins (UFT). Materiais e Métodos: O desenho do estudo é do tipo descritivo- transversal. A amostra foi composta por 38 idosos, sendo 28 do sexo feminino e 10 do sexo masculino. Foi aplicado um questionário para medir o conhecimento sobre saúde bucal dos idosos, analisando o índice CPOD e seus componentes. Resultados: O índice do CPOD foi de 57,89\%. Em relação às próteses, encontrou-se um percentual de 60,52\% usa prótese total e que os acadêmicos participantes da pesquisa $60,52 \%$ são endêntulos. No entanto, $93,42 \%$ dos idosos conhecem sobre autocuidado odontológico. Conclusão: A partir dos achados da pesquisa, pode-se concluir que o perfil da condição bucal dos idosos neste estudo é inadequado, porém, possuem alto índice de conhecimento de saúde bucal. Sugerimos a incorporação de programas de promoção de saúde e de reabilitação para este segmento da população.
\end{abstract}

Descritores: Idoso; Saúde Bucal; Universidades; Índice CPO.

\section{Abstract}

Introduction: The oral health of the elderly can be considered a public health problem. Objective: The study describes the oral health profile of the elderly at the University of Maturity (UMA) at the Palmas campus of the Federal University of Tocantins (UFT). Materials and Methods: The study design is descriptive-transversal. The sample consisted of 38 elderly people, 28 of whom were female and 10 were male. A questionnaire was applied to measure the knowledge about oral health of the elderly, analyzing the CPOD index and its components. Results: The CPOD index was $57.89 \%$. In relation to prostheses, a percentage of $60.52 \%$ was found to use total prosthesis and that the academics participating in the research $60.52 \%$ are endentulous. However, $93.42 \%$ of the elderly know about dental self-care. Conclusion: From the research findings, it can be concluded that the profile of the oral condition of the elderly in this study is inadequate, however, they have a high index of knowledge of oral health. We suggest the incorporation of health promotion and rehabilitation programs for this segment of the population.

Descriptors: Aged; Oral Health; Universities; DMF Index.

\section{Resumen}

Introducción: la salud bucal de los ancianos puede considerarse un problema de salud pública. Objetivo: El estudio describe el perfil de salud bucal de los ancianos en la Universidad de Madurez (UMA) en el campus de Palmas de la Universidad Federal de Tocantins (UFT). Materiales y métodos: el diseño del estudio es descriptivo-transversal. La muestra consistió en 38 personas mayores, 28 de las cuales eran mujeres y 10 hombres. Se aplicó un cuestionario para medir el conocimiento sobre la salud oral de los ancianos, analizando el índice DMFT y sus componentes. Resultados: El índice DMFT fue 57.89\%. En relación con las prótesis, se encontró que un porcentaje del $60.52 \%$ usa prótesis total y que los académicos que participan en la investigación del $60.52 \%$ son endentulous. Sin embargo, el $93.42 \%$ de las personas mayores sabe sobre el autocuidado dental. Conclusión: De los resultados de la investigación, se puede concluir que el perfil de la condición oral de los ancianos en este estudio es inadecuado, sin embargo, tienen un alto índice de conocimiento de la salud oral. Sugerimos la incorporación de programas de promoción y rehabilitación de la salud para este segmento de la población.

Descriptores: Anciano; Salud Bucal; Universidades; Índice de CPO.

INTRODUÇÃO

\section{O envelhecimento da população} brasileira apresentou um grande salto, quando comparado às últimas décadas. Segundo os dados do Instituto Brasileiro de Geografia e Estatística (IBGE) ${ }^{1}$, o Brasil possuía 21 milhões de pessoas $60+$ anos em 2012, correspondente a $12,6 \%$ do total da população brasileira. A estimativa da Organização Mundial da Saúde (WHO, 2005) ${ }^{2}$ indica que o país será o sexto maior em número de idosos em 2025, podendo chegar ao patamar de 32 milhões de pessoas com $60+$ anos ou mais.

A saúde dos idosos tem sido foco de muitos estudos, inclusive, na área odontológica, haja vista que a maioria desses idosos possui perda de dentes que acarretam em problemas consideráveis na mastigação, na articulação temporomandibular (ATM) e na vida social, estas condições estão associadas diretamente a sua saúde geral ${ }^{3}$. Doenças bucais aparecem de maneira desigual entre as pessoas em diferentes condições socioeconômicas, uma vez que as de baixa renda possuem uma pior saúde bucal, se comparada com aquelas que têm uma maior renda 4 .

O complexo da cavidade bucal é alterado com o envelhecimento. A perda de dentes é o principal problema enfrentado pelos idosos, esta por sua vez, acarreta em problemas na ATM, periodontites e gengivites com maior frequência, devido ao uso de próteses antigas ou mal adaptadas. Outro problema encontrado é a cárie radicular que está relacionada à falta de destreza manual 
para higienizar corretamente os dentes ${ }^{3}$. Por isso, maiores estudos são necessários para identificar os fatores que envolvem na saúde bucal dos idosos.

No entanto, no Brasil, estudos direcionados aos problemas bucais em idosos são escassos. A odontogeriatria se constituiu como um campo de atuação voltado para essas situações, promovendo assim, a inclusão por meio de tratamentos específicos para cada desordem bucal, além do acolhimento e atendimento humanizado para esses pacientes. Esse campo de atuação deve ser inserido em um contexto integral de atenção à saúde. Considerando a diversidade e a complexidade do idoso ${ }^{5,6}$.

Com vistas à elaboração de estratégias que promovam saúde bucal em idosos, tornouse necessário avaliar as condições socioeconômicas, clinicas e o conhecimento sobre cuidados odontológicos dos idosos. Portanto, esse artigo tem por objetivo descrever - perfil da saúde bucal dos idosos da Universidade da Maturidade do campus de Palmas - Tocantins, Brasil.

MATERIAL E MÉTODO

- Amostra

O estudo é do tipo descritivo, transversal com enfoque quantitativo. A amostra foi composta por indivíduos de ambos os sexos, sendo 28 do sexo feminino e 10 masculino, com idade $\geq$ a 60 anos, matriculados regularmente na UMA. Foram incluídos no estudo: aluno matriculado na UMA, com idade igual ou superior a sessenta anos. Para exclusão: estudante participante do estudo que se ausentou por algum motivo, bem como aqueles que não responderam aos questionários e ou não participaram das atividades de intervenção realizadas pelo estudo. O estudo foi aprovado pelo CEP/UFT sob o protocolo no 3.198.948.

\section{- Procedimentos}

Para obtenção das informações socioeconômicas foi realizada uma entrevista para aquisição dos dados sobre, sexo, renda e escolaridade. Para análise do conhecimento em saúde bucal, foi elaborado um questionário pelos autores do estudo. O questionário foi composto por 16 perguntas de múltipla escolha, com temas sobre cárie, placa bacteriana, tártaro, prevenção, higienização, autocuidado e flúor. Posteriormente, foi avaliada a condição clínica de cada idoso e para isso foi utilizado o instrumento CPOD, composto pelos seguintes fatores: dentes cariados $(C)$, perdidos $(P)$, e obturados (O). O exame clínico seguiu critérios do manual do Ministério da Saúde que considera o dente cariado, quando há evidência clínica de esmalte socavado, existindo uma cavidade definida com descoloração ou opacidade ao longo das margens. O dente foi considerado "obturado", quando se observou a reconstituição da cavidade com material restaurador direto ou indireto. Dentes com lesão de cárie secundária foram classificados como cariados. O índice CPOD, formulado por Klein e Palmer ${ }^{7}$, em 1937, é usado pela Organização Mundial de Saúde (OMS) para avaliar a prevalência da cárie dentária em diversos países. A sigla "CPO tem origem nas palavras cariados, perdidos e obturados" e o D indica que a unidade de medida é o dente. Por fim, foi realizada uma anamnese para verificação das condições das próteses dentárias dos idosos. Nas próteses removíveis sejam totais ou parciais foram considerados os seguintes tópicos: a) uso de prótese total ou parcial removível no momento do exame; b) próteses com mais de cinco anos de uso foram classificadas como inadequadas, assim como se estivessem faltando dentes, quebradas ou folgadas.

- Analise dos dados

Os dados obtidos por meio dos questionários foram tabulados no programa Excel, analisados e apresentados com a estatística descritiva.

RESULTADOS E DISCUSSÃO

As informações socioeconômicas estão apresentadas nas Tabelas 1, 2 e 3 . Com uma maior participação das mulheres e com idade entre 65 a 69 anos. Em relação às questões econômicas, concentrando o maior número dos entrevistados, a renda familiar foi considerada baixa, pois varia de $R \$ 477,00$ a $R \$ 1.908,00$ reais. Segundo Brunetti et al. $^{8}$, fatores ambientais como baixa renda e acesso reduzido aos cuidados dentários, também têm sido implicados na gênese da cárie em idosos. Podese considerar que a cárie é um fenômeno mundial nas populações idosas, com fatores predisponentes: higiene oral inadequada, acúmulo de placa, uso de dentaduras, inadequada exposição a flúor, dentre outros8. Em relação à escolaridade, os questionários apontaram que a maioria dentre os entrevistados possui somente 0 ensino fundamental e o ensino médio, ou seja, baixa escolaridade. Estudos demonstraram que a baixa escolaridade, pode provocar maior incidência de edentulismo ${ }^{9,10}$.

As perdas dentárias podem ser consideradas um problema de saúde pública, uma vez que o tratamento possui um alto custo, 
assim, a ocorrência do edentulismo incide nas classes mais desfavorecidas da população. 0 uso da prótese dentária "é indicado para a recuperação da capacidade mastigatória, para a melhora do aspecto estético e de fonação dos indivíduos acometidos pela perda dental, impactando na qualidade de vida"11.

Tabela 1. Idade dos participantes da pesquisa, Palmas/TO Brasil, 2020

\begin{tabular}{|c|c|c|c|}
\hline Idade (anos) & Mulheres & Homens & $\%$ total \\
\hline 60 a 64 anos & $23,68 \%$ & $7,89 \%$ & $31,57 \%$ \\
\hline 65 a 69 anos & $31,58 \%$ & $0 \%$ & $31,58 \%$ \\
\hline 70 a 74 anos & $7,89 \%$ & $5,26 \%$ & $13,15 \%$ \\
\hline$>75$ anos & $10,53 \%$ & $13,16 \%$ & $23,68 \%$ \\
\hline
\end{tabular}

Tabela 2. Renda dos participantes da pesquisa, Palmas/TO Brasil, 2020

\begin{tabular}{|c|c|c|c|}
\hline Renda Familiar & Mulheres & Homens & $\%$ total \\
\hline De 477,00 a 954,00 & $23,69 \%$ & $5,26 \%$ & $28,95 \%$ \\
\hline De 954,00 a 1.908,00 & $23,69 \%$ & $10,53 \%$ & $34,22 \%$ \\
\hline De 1.908,00 a $2.862,00$ & $7,89 \%$ & $2,63 \%$ & $10,52 \%$ \\
\hline De 2.862,00 a 3.816,00 & $13,16 \%$ & $5,26 \%$ & $18,42 \%$ \\
\hline De 3.816,00 a 4.770,00 & $2,63 \%$ & $0 \%$ & $2,63 \%$ \\
\hline De 4.770,00 a 5.724,00 & $0 \%$ & $2,63 \%$ & $2,63 \%$ \\
\hline Acima de 5.724,00 & $2,63 \%$ & $0 \%$ & $2,63 \%$ \\
\hline
\end{tabular}

Fonte: Questionário aplicado (2020).

Tabela 3. Nível de escolaridade dos partícipes da pesquisa, Palmas/TO - Brasil, 2020

\begin{tabular}{|c|c|c|c|}
\hline Anos de Estudo & Mulheres & \multicolumn{1}{c|}{ Homens } & $\%$ total \\
\hline $1-4$ anos & $36,84 \%$ & $10,53 \%$ & $47,37 \%$ \\
\hline $5-8$ anos & $23,68 \%$ & $7,89 \%$ & $31,57 \%$ \\
\hline $9-11$ anos & $7,89 \%$ & $5,26 \%$ & $13,15 \%$ \\
\hline$>11$ anos & $2,63 \%$ & $0 \%$ & $2,63 \%$ \\
\hline Fon
\end{tabular}

Fonte: Questionário aplicado (2020)

Estudo realizado por Azevedo et al. ${ }^{11}$, com um total de 7.496 idosos apontam que a prevalência de próteses é de $78,2 \%$, para o uso e a necessidade de prótese é de $68,7 \%$. Dentre as regiões brasileiras, a região norte, onde se localiza o Estado do Tocantins, é a região de dados mais elevados $76,2 \%$ e $80,6 \%$ (prevalência e necessidade respectivamente). Neste sentido, a situação da saúde bucal dos idosos da UMA, assemelha-se com o que foi relatado na literatura ${ }^{11}$, onde 0 nível de escolaridade $e$ as questões financeiras influenciam no tratamento e na saúde bucal dos idosos.

Nas tabelas 4, 5, 6, 7 são apresentadas as respostas dos idosos em relação ao nível de conhecimento sobre saúde bucal. As tabelas foram divididas por assunto, sendo a tabela 4 (autocuidado), 5 (cárie) e 6 (prevenção, flúor, escovação, fio dental) e 7 (gengivite). Foi encontrado um ótimo conhecimento, pois, as porcentagens de acertos variaram entre $60,53 \%$ (menor acerto) a 94,74\% (maior acerto).

Os acadêmicos que demonstraram conhecer sobre as necessidades do autocuidado tiveram uma média de acertos de $93,42 \%$. Matos e Lima-Costa ${ }^{12}$ observaram, em sua pesquisa, que dentre os entrevistados com indicação para tratamento reabilitador, aqueles não usuários de prótese apresentaram uma chance $13 \%$ menor de relatar uma saúde bucal "ótima" ou "boa" em relação aos 49 usuários de próteses.

Tabela 4. Autocuidado odontológico, Palmas/TO - Brasil, 2020

\begin{tabular}{c|c|c} 
Variável & Erros (\%) & Acertos (\%) \\
Q7 & 5,26 & 94,74 \\
\hline Q10 & 2,63 & 97,37 \\
\hline Q11 & 15,79 & 84,21 \\
\hline Q12 & 2,63 & 97,37 \\
\hline Média Total & 6,58 & 93,42 \\
\hline \multicolumn{2}{l}{ Fonte: Questionário aplicado (2020). }
\end{tabular}

A análise clinica efetuada nos idosos apresentou um percentual de 18,42\% com cárie, um dado preocupante, e, analisando que $60,53 \%$ possuem dentes obturados, certificouse que a problemática era muito maior, pois tiveram a cárie e a mesma foi tratada. A situação agravou-se dentre os participantes da pesquisa com a média de $94,74 \%$ de dentes perdidos. A perda pode ter sido por cárie não tratada, ou por falta de recursos financeiros para tratamentos no geral, acarretando a extração total. Essa perda gerou a necessidade de tratamento reabilitador.

Tabela 5. Participantes respondem sobre seu conhecimento em relação à Cárie, Palmas/TO - Brasil, 2020

\begin{tabular}{c|c|c} 
Variável & Erros (\%) & Acertos (\%) \\
\hline Q1 & 26,32 & 73,68 \\
\hline Q6 & 21,05 & 78,95 \\
\hline Q8 & 2,63 & 97,37 \\
\hline Média Total & 16,67 & 83,33 \\
\hline \multicolumn{2}{l}{ Fonte: Questionário aplicado (2020). }
\end{tabular}

Os acadêmicos tiveram uma média de $83,33 \%$ de acertos em relação a três perguntas sobre cárie. A cárie é ainda uma das doenças bucais que mais acomete aos brasileiros, apesar de existirem tratamentos eficazes para combatê-la. Ela possui característica invasiva e destrutiva, acarretando problemas mais sérios ou até mesmo a perda do elemento dental se não tratada a tempo. Adicionalmente, os valores elevados do índice de cárie indicam hábitos nocivos da população, decorrentes da falta de conhecimento e conscientização, como alta frequência de ingestão de açúcar e escovação inadequada, e apontam, também, a dificuldade de acesso aos serviços de saúde preventivos e curativos $^{13-16}$.

Tabela 6. Prevenção, flúor, escovação, fio dental, Palmas/TO Brasil, 2020

\begin{tabular}{|c|c|c|}
\hline Variável & Erros (\%) & Acertos (\%) \\
\hline Q3 & 26,32 & 73,68 \\
\hline $\mathrm{Q}_{4}$ & 13,16 & 86,84 \\
\hline Q15 & 28,95 & 71,05 \\
\hline Q16 & o & 100 \\
\hline Média Total & 17,10 & 82,90 \\
\hline
\end{tabular}

Os dados referentes à tabela 06 apontaram que a média total de acertos dos pesquisados foi de $82,90 \%$, ou seja, demonstraram conhecer adequadamente sobre 
escovação, flúor, prevenção e uso de fio dental. Existem algumas evidências de associação entre autoavaliação de saúde bucal e indicadores da condição dentária e periodontal. Por outro lado, estudos têm descoberto percepções positivas de saúde bucal entre indivíduos com grande número de dentes perdidos ou com uma condição periodontal precária, sugerindo um padrão de avaliação diferente dos padrões clínicos (normativos) de saúde bucal ${ }^{17,18}$. A percepção de saúde bucal é diretamente afetada pela habilidade para mastigar, satisfação com a aparência e pela ausência de dor, apresentando pouca relação com a avaliação clínica da condição bucal ${ }^{19}$.

Segundo Ávila et al. ${ }^{20}$, a cavidade bucal reflete as alterações do envelhecimento. Dos problemas bucais existentes nos idosos, a perda de dentes é um dos mais frequentes. A perda da dentição permanente influencia na mastigação, digestão, gustação, pronúncia e na estética. Dentre as alterações ocorridas na cavidade oral do idoso, a perda de elementos dentários é a que implica em maiores consequências para os demais órgãos do corpo humano. Essa perda pode ser ocasionada, também, por uma gengivite não tratada. $O$ estudo apontou que $60,52 \%$ dos acadêmicos são edêntulos, e em relação à compreensão sobre gengivite, demonstraram conhecer. A pesquisa não reconheceu, especificamente, a causa de $94,74 \%$ das perdas de dentes.

Tabela 7. Conhecimento sobre Gengivite, Palmas/TO - Brasil, 2020

\begin{tabular}{c|c|c}
\hline Variável & Erros (\%) & Acertos (\%) \\
\hline Q5 & 5,26 & 94,74 \\
\hline Q13 & 21,05 & 78,95 \\
\hline Q14 & 2,63 & 97,37 \\
\hline Média Total & 9,65 & 90,35 \\
\multicolumn{2}{l}{ Fonte: Questionário aplicado (2020). }
\end{tabular}

$\mathrm{Na}$ tabela 8, é apresentado o índice CPOD dos idosos. Os participantes da pesquisa na UMA, mesmo demonstrando conhecimento sobre o assunto, não possuíam a mesma correspondência na realidade vivenciada, pois a média geral no CPOD foi de $57,89 \%$. São várias as evidências que apontam que 0 cuidado odontológico regular possibilita um estado de saúde bucal mais satisfatório e melhor percepção. Por isso, sugerimos maiores investimento em programas de promoção e prevenção de saúde bucal ${ }^{17,21,22}$.

Tabela 8. Índice CPOD e seus componentes, Palmas/TO - Brasil, 2020

\begin{tabular}{|c|c|c|c}
\hline CPOD e seus componentes & $\mathbf{N}$ & $\%$ & Média \\
\hline Cariado & 10 & 1,32 & $18,42 \%$ \\
\hline Perdido & 614 & 81 & $94,74 \%$ \\
\hline Obturado & 134 & 17,68 & $60,53 \%$ \\
\hline Total & 758 & 100 & \\
\hline
\end{tabular}

Fonte: Questionário aplicado (2020).
$\mathrm{Na}$ tabela 9 são apresentados dados da anamnese clínica da condição das próteses. Se $60,52 \%$ usavam prótese total, ou seja, perderam os dentes, $34,21 \%$ possuíam dentes parcialmente, e 5,26\% não faziam uso de nenhuma prótese. A partir desses dados, percebeu-se que o uso da prótese oportunizou aos pesquisados uma melhor qualidade de vida na questão da mastigação, e autoestima.

Constatou-se uma melhor percepção de saúde bucal entre os indivíduos com maior escolaridade e com maior renda, e, de maneira geral, todos demonstraram possuir bons conhecimentos sobre saúde bucal. Para mais, Gift et al. ${ }^{17}$, Heft et al. ${ }^{23}$, John et al. ${ }^{24}$ e Tassinari et al. $^{25}$, encontraram uma percepção mais positiva da saúde bucal em indivíduos com mais anos de estudo.

Em relação ao estudo realizado com o grupo idosos da UMA constatou-se que $60,52 \%$, ou seja, a maioria são edêntulos, os demais do grupo usavam prótese parcial e possuíam baixa escolaridade (Tabela 3). Esse estudo coaduna com as informações dos autores citados anteriormente. Para Cruz et al. ${ }^{26}$, condições econômicas e de saúde ao longo da vida, bem como bem-estar psicológico de populações diversas têm efeitos cumulativos diferenciais na saúde bucal e na qualidade de vida relacionada a ela.

Tabela 9. Uso da prótese pelos pesquisados. Distribuição dos idosos segundo a condição de prótese (satisfatória ou insatisfatória). Palmas/TO - Brasil, 2020

\begin{tabular}{|c|c|c|}
\hline Condição da Prótese Dentária & $\mathbf{N}$ & $\%$ \\
\hline \multicolumn{3}{|l|}{ Prótese Total } \\
\hline Satisfatória & 8 & 34,78 \\
\hline Insatisfatória & 15 & 65,22 \\
\hline \multicolumn{3}{|l|}{ Prótese Parcial Removível } \\
\hline Satisfatória & 7 & 53,85 \\
\hline Insatisfatória & 6 & 46,15 \\
\hline
\end{tabular}

Fonte: Questionário aplicado (2020).

Segundo Brunetti et al. ${ }^{27}$, a ausência parcial ou total de dentes e o uso de próteses inadequadas levam a uma redução na capacidade mastigatória, deixando o indivíduo de ingerir nutrientes essenciais em sua dieta, contribuindo para exacerbar os problemas sistêmicos que porventura possam estar se apresentando e comprometendo a saúde geral.

$\mathrm{Na}$ tentativa de correção, visando melhoria estética e de mastigação, busca-se nas próteses minimizar a situação encontrada do edentulismo. No estudo realizado na Universidade da Maturidade, em relação às próteses, encontrou-se o maior número de próteses insatisfatórias em relação às condições de higiene, tempo de uso da prótese sem manutenção e conservação. Quanto às próteses parciais removíveis, apresentou-se 
maior número em condições satisfatórias. Podese considerar que a maioria se enquadrou na situação do endentulismo 60,52\% (ausência completa de elementos dentários).

Para Mcgrath e Bedi ${ }^{28}$, constataram o efeito mediador do uso de prótese, encontrando maior relato de impactos na qualidade de vida entre as pessoas que faziam uso de prótese. Os dados encontrados na UMA foram em relação ao número de prótese, ou seja, houve um quantitativo de 23 próteses totais (podendo ser arcada inferior e superior) e 13 próteses parciais, as análises foram efetuadas computando a quantidade de próteses e não de indivíduos participantes da pesquisa.

No que se refere à saúde bucal, existe o ideal e o real. $O$ ideal seria as pessoas poderem ir ao dentista regulamente, mas o real, devido ao alto custo, busca-se o tratamento dentário, quando não há outra saída. $E$, historicamente falando, era mais fácil extrair um dente, do que tratá-lo. A saúde bucal na terceira idade é um fator essencial para o envelhecimento saudável e uma boa qualidade de vida. No entanto, as condições desiguais em que as pessoas vivem e trabalham são refletidas nitidamente na saúde bucal, uma vez que idosos expostos às situações de vulnerabilidade social estão mais sujeitos à interferência direta dos determinantes sociais no processo saúde-doença.

\section{CONCLUSÃO}

O objetivo central do estudo foi o de descrever o perfil da saúde bucal dos idosos da Universidade da Maturidade do campus de Palmas. Os idosos da UMA possuem um alto conhecimento de saúde bucal. No entanto, possuem baixas condições de saúde bucal, quando analisamos CPOD e condição de prótese. Dentre os fatores para essas condições encontramos baixa escolaridade e renda. A estratégia para mudar a realidade que prevalece atualmente é propor programas de prevenção e cuidado de saúde bucal. Sugerimos uma intervenção odonto pedagógica, sendo esta, uma nova proposta de ação dentro da Instituição.

\section{REFERÊNCIAS}

1. IBGE-Instituto Brasileiro de Geografia e Estatística - IBGE (2013). Síntese dos indicadores sociais, estudos e pesquisas. Rio de Janeiro.

2. Organização Mundial da Saúde (OMS). Envelhecimento ativo: uma política de saúde. Brasília: Organização Pan-Americana de Saúde, 2005.

3. Presa SL, Matos JC. Saúde bucal na terceira idade, Rev UNINGÁ. 2014;39:137-48.
4. Vettore MV, Aqeeli A. The roles of contextual and individual social determinants of oral health-related quality of life in Brazilian adults. Qual Life Res. 2016;25(4):1029-42.

5. Barbosa KGN. Condições de saúde bucal em idosos: uma revisão da realidade brasileira. Odontol Clín.-Cient. 2011;10(3):227-31.

6. Rosa LB, Zuccolotto MCC, Bataglion C, Coronatto Z. A. Odontogeriatria - a saúde bucal na terceira idade. RFO. 2008;13(2):82-6.

7. Klein H, Palmer CE. Dental caries in American Indian children. Public Health Bull. 1937; 239:1-53.

8. Brunetti R, Montenegro F, Marchini L. Odontogeriatria - uma visão gerontológica. Rio de Janeiro: Elsevier; 2013.

9. Brondani MA. Educação preventiva em odontogeriatria, mais do que uma necessidade, uma realidade. Rev odonto ciênc. 2002;17(35): 57-61.

10. Brunetti R, Montenegro F. Odontogeriatria: noções de interesse clínico. São Paulo: Artes Médicas; 2002.

11. Azevedo JS, Azevedo MS, De Oliveira LJC, Correa MB, Demarco FF. Uso e necessidade de prótese dentária em idosos brasileiros segundo a Pesquisa Nacional de Saúde Bucal (SBBrasil 2010): prevalências e fatores associados. Cad Saúde Pública 2017;33(8): e00054016.

12. Matos DL, Lima-Costa MF. Auto-avaliação da saúde bucal entre adultos e idosos residentes na Região Sudeste: resultados do Projeto SBBrasil 2003. Cad Saúde Pública 2006;22(8): 1699-707.

13. Burt BA. Fifty years of water fluoridation. Br. Dent J. 1995;178(2):49-50.

14. Locker D. Impact of dental conditions on patient's quality of life. Community Dent Health. 1997;5(1):3-18.

15. Pereira AC. Odontologia em Saúde Coletiva: planejando ações e promovendo Saúde. Porto Alegre: Artmed; 2003.

16. Peres MA, Peres KG, Barros AJD. The relation between family socioeconomic trajectories from childhood to adolescence and dental caries and associated oral behaviours. J Epidemiol Commnunity Health. 2007;61(2):141-45.

17. Gift HC, Atchison KA, Drury TF. Perceptions of the Natural Dentition in the Context of Multiple Variables. J Dent Res. 1998;77(7):1529-38.

18. Afonso-Souza G, Nadanovsky PCD, Faerstein E, Werneck GL, Lopes CS. Association between routine visits for dental checkups and self-perceived oral health in an adult population in Rio de Janeiro: the pró-saúde study. Community Dent Oral Epidemiol. 2007;35: 393-400.

19. Matthias RE, Atchison KA, Lubben JE, De JONG F, Schweitzer SO. Factors affecting selfratings of oral health. J Public Health Dent. 
1995;5(4):197-204.

20. Ávila LX, De Carli JP, Corralo D, Trntin MS. Extensão Universitária como mediadora da saúde bucal do idoso. Revista de Extensão universitária de Cruz Alta. Cataventos. 2018; 10(1):15-32.

21. Andersen RM, Davidson PL. Ethnicity, aging, and oral health outcomes: a conceptual framework. Adv Dent Res. 1997;11(20):3-9.

22. Maupomé $G$, Peters D, White A. Use of clinical services compared with patients perceptions of and satisfaction with oral health status. J Public Health Dent. 2004;64(2):88-95.

23. Heft MW, Gilbert GH, Shelton BJ, Duncan RP. Relationship of dental status, sociodemographic status, and oral symptoms to perceived need for dental care. Community Dent Oral Epidemiol. 2003;31(3):51-60.

24. John MT, Koepsell TD, Hujoel P, Miglioretti DL, Leresche L, Micheelis W. Demographic factors denture status and oral health-related quality of life. Community Dent Oral Epidemiol 2004;32: 125-32.

25. Tassinari WS. Contexto sócio-econômico e percepção da saúde bucal em uma população de adultos no Rio de Janeiro, Brasil: uma análise multinível. Cad Saúde Pública. 2007; 3(1):127-36.

26. Cruz DT, Caetano VC, Leite, ICG. Envelhecimento populacional e bases legais da atenção à saúde do idoso. Cad saúde colet. 2010;18(4):500-8.

27. Brunetti R, Montenegro F, Marchini L. Odontogeriatria - uma visão gerontológica. Rio de Janeiro: Elsevier, 2013.

28. Mcgrath C, Bedi R. Can dentures improve the quality of life of those who have experienced considerable tooth loss? J Dent. 2001;29: 243-46.

\section{CONFLITO DE INTERESSES}

Os autores declaram não haver conflitos de interesse

\section{AUTOR PARA CORRESPONDÊNCIA}

\author{
Eduardo Aoki Ribeiro Sera \\ Universidade Federal do Tocantins - UFT \\ 77001-090 Palmas-TO, Brasil \\ E-mail: eduardosera@live.com
}

Submetido em 01/08/2020

Aceito em 23/10/2020 